\title{
The Sphericity Effects in Model Atmospheres of Central Stars of planetary nebulae
}

\section{Jiří Kubát}

\section{Astronomický ústav, 25165 Ondřejov, Czech Republic}

The model atmospheres of central stars of planetary nebulae are often calculated under the assumption of the plane-parallel geometry. This assumption seems to be reasonable for stars with relatively thin atmospheres like white dwarfs, where the thickness of the atmosphere is only few kilometers while the corresponding radius is about several thousands of kilometers. Nevertheless, calculations of a grid of pure hydrogen model atmospheres of hot white dwarfs demonstrated that this assumption may fail even for thin atmospheres (Kubát 1995). We found small differences between line profiles (about 1\%) calculated under the assumption of plane-parallel and spherically symmetric atmospheres. Such differences are detectable by contemporary observational technique, and, consequently, may not be neglected. In addition, sphericity effects are important also in stars of other types (Kubát 1996). We decided to test the assumption of the plane parallel geometry also for sample model atmospheres of central stars of planetary nebulae. We assumed static atmospheres in radiative, hydrostatic, and statistical equilibrium (NLTE) consisting of hydrogen and helium. We calculated spherically symmetric and plane parallel model atmospheres of two central stars, namely LoTr4 and K1-27. Basic atmospheric parameters of these stars were adopted from Rauch et al. $(1996,1994)$, respectively. The atmospheric parameters for LoTr4 $\left(T_{\text {eff }}=120000 K, \log g=5.5, M=0.65 M_{\odot}, n(H) / n(H e)=0.5\right.$ yield a model with an extension $\left(r\left(\tau_{R}=10^{-5}\right) / r\left(\tau_{R}=2 / 3\right)\right)$ of 1.028 . For K1-27, the atmospheric parameters $\left(T_{\text {eff }}=100000 K, \log g=6.5, M=0.52 M_{\odot}, n(H) / n(H e)=\right.$ 0.2 produce only a small extension of 1.0035 . The differences in temperature structure of our model atmospheres are more pronounced for a star with lower gravity and, consequently, larger extension. Continuum flux is lower for spherical atmospheres. This difference is larger for LoTr4, i.e. a star with more extended atmosphere, and almost negligible for K1-27. Paschen and Pickering He II lines also show differences. These differences are quite large (several per cent) for $\alpha$ lines, and they decrease towards higher series members. Using plane-parallel model atmospheres instead of spherically symmetric ones introduces a systematic error into results. This error is present also in highly sophisticated NLTE line blanketed models. Due to the above mentioned differences one should avoid using lower series members (e.g. He II $4686 \AA$ ) for determination of atmospheric parameters $\left(T_{\mathrm{eff}}\right.$, $\log g$, abundances, ...) from plane-parallel atmospheres. Details of our calculations will be presented in Astronomy and Astrophysics.

This work was supported by grants C3003601 and 205/96/1198, and by projects K1003-601/4 and K1-043-601.

\section{REFERENCES}

Kubát J., 1995, A\&A, 299, 803

Kubát J., 1996, A\&A, 305, 255

Rauch T., Köppen J., Werner K., 1994, A\&A, 286, 543

Rauch T., Köppen J., Werner K., 1996, A\&A, 310, 613 